\title{
2 The Use of Computer Programs for Analyzing Suicide Texts
}

The vast majority of previous research on texts written by suicides has focused on suicide notes, and most of this research on the content of suicide notes has used judges to identify the presence or absence of content in the note. This requires subjective judgments which can be validated by looking at the inter-judge agreement between the two or more judges used. The advantage of this technique is that the researchers can decide what content categories are most relevant to their hypotheses, and they can have their judges score the content for these categories. In contrast, computer programs are typically devised to score texts for predetermined categories which may or not be pertinent to the researchers' hypotheses. Computer analyses tend, therefore, to be purely empirically-oriented rather than theoretically-oriented.

Several earlier studies have used computer programs to analyze suicide notes. Ogilvie, et al. (1965) compared a sample of genuine and simulated notes written by men and found that the genuine notes had more references to "things" such as roles and objects, whereas the simulated notes had more references to emotional states. Ogilvie, et al. also carried out more detailed analyses They examined sentences referring to the female role and found that the writers of genuine notes gave fewer instructions to females, gave more information about females, and made more references to being acted upon by females than the writers of simulated notes. Looking at the instructions given to females, the genuine note writers gave more specific instructions and fewer vague instructions. Looking at actions, the genuine note writers used the word "think" in the context of knowing or decision making (I think that if I went to the doctor, I would.....), whereas the simulated note writers used the concept more in the context of solving a problem (I am thinking of all the problems we have shared). Using three items (references to concrete things, places and persons; the use of the word love in the text; and the total number of references to processes of thought and decisions), Ogilvie, et al. were able to correctly classify 30 of the 33 pairs of genuine and simulated notes. Of course, this result needs to be replicated on a new set of genuine and simulated suicide notes to check on its reliability.

Henken (1976) also compared genuine and simulated suicide notes and notes written by people about to die using a computer program. The genuine notes were more concrete, constricted and concerned with interpersonal relationships (especially those with people of the opposite sex). Edelman and Renshaw (1982), using computer analysis, found that the genuine notes were longer and had fewer positive modifiers, more negative existential density (e.g., "not”), more negative authority (e.g., proper nouns), more negative audience (e.g., "you”), more modified nouns, more modified verbs, more negative generalized others (e.g., third-person pronouns), more cognizance of objects (concrete objects), more modification of objects and actions, more negative modification of unknown people, more static action, and fewer references to 
future time. To be honest, most of the differences identified seem to me to have little psychodynamic relevance.

The present set of studies uses a computer program (the LIWC; Pennebaker, et al., 2001) that, while still lacking a theoretical basis, does score texts for interesting content. The program has been used in a variety of studies of texts so that there is a body of research already published using the program. Many of the chapters in this book use the LIWC to study suicide notes, letters and blogs written by suicides, diaries from suicides, poems written by poets who died by suicide, and the words used in tapes recordings from suicides. The studies include both cross-sectional studies (such as comparing the suicide notes of men and women) and time-series studies (such as how the content of a diary changes over time.

\subsection{The Linguistic Inquiry Word Count (LIWC)}

The LIWC has been used to analyze many documents, including the narratives online by women with breast cancer (Bantum \& Owen, 2009), the value of written essays about trauma in helping trauma victims cope (Klest \& Freyd, 2007), the differences between truthful and deceptive language in prisoners (Bond \& Lee, 2005), the differences between essays written by psychiatrically disturbed and normal individuals (Junghaenel, et al., 2008), personality differences in self-narratives (Hirsh \& Peterson, 2009), and differences in the interviews between writers and physicists (Djikic \& Oatley, 2006). There are also a large number of, as yet unpublished, doctoral dissertations that have used the LIWC. The LIWC has been adapted for use into Dutch (Zijlstra, et al., 2004), German (Wolf, et al., 2008), Korean (Lee, et al., 2006) and Spanish (Ramirez-Esparza, et al., 2007).

\subsection{The Use of the LIWC for the Study of Suicide}

Prior to this set of studies on texts from suicides, several studies have appeared using the LIWC. Pennebaker and Stone ((2004; see Chapter 3$)$ studied the diary of a young women who completed suicide and found that the proportion of words indicating positive emotions increased as Katie came closer to the time of her suicide while the proportion of words indicating negative emotions decreased. Religious words increased while words related to death decreased.

Williams (2007) compared suicide notes from attempted and completed suicides and found no differences in words indicating belongingness and burdensomeness. In diaries, words indicating belongingness did not change over time, but words indicating optimism, certainty and causality increased as the suicide approached. Suicidal students did use more words indicating burdensomeness in their essays as compared to nonsuicidal students, but did not differ in words indicating belongingness. Also 
comparing suicide notes from attempted and completed suicides, Handelman and Lester (2007) found that the notes from completed suicides had fewer metaphysical references, more future tense verbs, more social references to others, and more positive emotions than did the notes from attempted suicides.

Stirman and Pennebaker (2001) compared the poems of nine poets who completed suicide with those of nine poets who did not do so. They differed in words indicating hopelessness and integration. Regarding hopelessness, it was predicted that the suicides would have more words indicating negative emotions and death and fewer words indicating positive emotions. Only the difference in death-related words was significant. Regarding integration, it was predicated that the suicides would have more self-references and fewer references to communication with others. The suicides did have more first-person singular words (e.g., me and my), but no differences in references to others, first-person plural words (e.g., we) or communications words. Over time, the suicides used first-person plural words less often. Although it was not predicted, the suicides did use more sex-related words throughout their poems than did the comparison poets.

Rogan (2009) studied the audio-transcriptions of words used in four crisis negotiation incidents. Two perpetrators surrendered to the police, while two completed suicide after releasing hostages. Several significant differences were identified. In the suicide incidents, there was fewer words related to anger, inhibition, seeing, and physical being. Predictions made on the basis of clinical knowledge about suicides (such as words indicating positive emotion, sadness and depression and personal pronouns) were not confirmed.

More recently, Stack, et al. (2012) compared suicide songs written by twenty four groups (such as Metallica and 3 Doors Down) with the neighboring track on the CD using the LIWC. The suicide songs were quite similar in content to the comparison songs except for two differences: the suicide songs had more words per sentence and a higher percentage of sad words, but they did not differ in other emotions or in references to death and suicide. Stack et al. concluded that the great degree of similarity explains why research exposing individuals to suicide songs has found no impact on their suicidal ideation, and why suicide songs per se do not appear to precipitate suicidal behavior. Any impact of the music of a group appears to result from the overall style and content of the group's music and not to any particular song.

Lester (2012) used the LIWC to compare the written memorials on online bereavement websites written by those who have lost a significant other to suicide and those who have lost a significant other from other causes. He found that the memorials written by suicide survivors were longer (and used longer words). They had more references to death, fewer references to themselves or to the deceased, and more words reflective of anger and sadness. He concluded that deaths from suicide have a more profound impact on the survivors and result in greater emotional distress than do natural deaths. 
Many of the chapters, especially on those on the diaries left by those who died by suicide, use my subjective, qualitative interpretation. For the published diaries, readers can obtain a copy of the books and explore their own interpretation of the psychodynamics of the writer. For the personal diaries I have obtained from their significant others, only Katie's diary is available. The last month of this diary appears in Lester (2004b). I do not have permission to share the diaries of Jim (Chapter 6) or Robert (Chapter 7).

I use the LIWC in many of the following chapters, but I report only the significant trends and differences that I identified. The complete list of variables measured by the LIWC is shown in Table 2.1. Apart from the word count and the words per sentence, the other measures are expressed as percentages. For example, the measure for anger is the percentage of words expressing anger relative to the total word count. It is possible, of course, for a particular word to fit into two or more categories. For example, a swear word may also be a sexual word. The results of the LIWC analyses in each chapter are usually presented as Pearson correlation coefficients for ease of interpretation. Correlation coefficients can go from -1.0 to +1.0 and are often used as a measure of effect size.

Tab. 2.1: The LIWC categories, with examples of words.

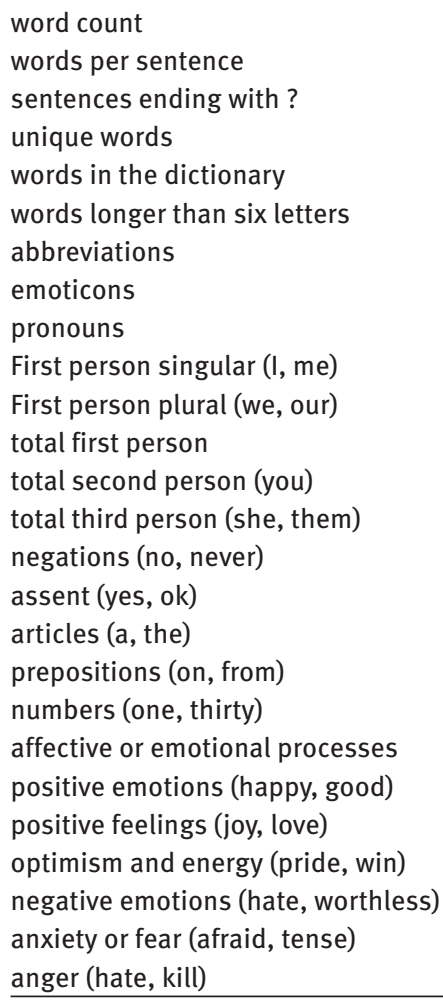


continued Tab.2.1.: The LIWC categories, with examples of words.

sadness or depression (cry, sad)

cognitive processes

causation (because, hence)

insight (think, know)

discrepancy (should, would)

inhibition (block, constrain)

tentative (maybe, perhaps)

certainty (always, never)

sensory and perceptual processes

seeing (saw, view)

hearing (heard, listen)

feeling (touch, hold)

social processes

communication (talk, share)

other references to people (pronouns)

friends (pal, buddy)

family (brother, father)

humans (boy, woman)

time (hour, day)

past tense verb

present tense verb

future tense verb

space (around, over)

up (above, over)

down (below, under)

inclusive (with, include)

exclusive (but, except)

motion (walk, move)

occupation (work, boss)

school (class, student)

job or work (boss, career)

achievement (goal, win)

leisure activity

home (house, kitchen)

sports (football, game)

television and movies (TV, sitcom)

music (song, CD)

money and financial issues (cash, taxes)

metaphysical issues (God, heaven)

religion (church, rabbi)

death and dying (dead, coffin)

physical states and functions

body states, symptoms (heart, cough)

sex and sexuality (penis, fuck)

eating, drinking, dieting (eat, swallow)

sleeping, dreaming (asleep, bed)

grooming (wash, clean)

swearing (fuck, damn) 